\title{
Introduction to the special issue on service-oriented E-business development
}

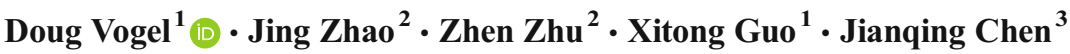

Received: 19 May 2017 / Accepted: 22 May 2017 / Published online: 2 June 2017

(C) The Author(s) 2017. This article is an open access publication

\section{Introduction to the special issue on service-oriented E-business development}

We are witnessing a transition from a goods oriented economy to a services-oriented economy. Service has become increasingly prevalent and important for E-business development in the world economy, especially for emerging economies. As a firm attempts to move from goods to services, managers should rethink service-oriented E-business strategies and operations through transiting to services management for their portfolio of resources and capabilities. Business practices have shown new ways and innovative behaviors in IS usage, marketing development, and service operations for improving service-orientated E-business. It provides opportunities as well as challenges for E-business practices and research. Service-oriented E-business best practices, standards, processes, security, big data analytics, computing formalisms, business models, etc. are important issues that warrant exploration.

Doug Vogel

isdoug@hit.edu.cn

Jing Zhao

zhao5563@gmail.com

Zhen Zhu

zhuzhen2008@gmail.com

Xitong Guo

xitongguo@hit.edu.cn

Jianqing Chen

chenjq@utdallas.edu

Harbin Institute of Technology, Harbin, China

2 China University of Geosciences, Beijing, China

3 University of Texas at Dallas, Richardson, TX, USA
The purpose of this special issue is to bring together innovative research in the areas of service engineering, operations management and marketing design to develop cumulative knowledge as represented in the three accepted papers.

- A Computer-based Approach for Analyzing Consumer Demands in Electronic Word of Mouth (by Yuh-Min Chen, Chung-Yi Lin, Mao-Yuan Pai, Chao-Chun Chen and Shu-Yi Lia) explores consumer opinions as one of the most valuable assets that enterprises have when consumers share their appraisals of products or services through electronic word-of-mouth (eWOM). Since these consumer appraisals usually reflect consumer needs, and thus their demands, collecting and analyzing eWOM data has become a key task for many businesses. In order to effectively collect and analyze eWOM data, this study proposes a computer-based approach for analyzing consumer demands. It is anticipated that companies will be able to improve their products and services through the application of this computer-based approach, thus enhancing their competitiveness. This study has the following objectives: (i) constructing a consumer demands analysis model in eWOM; (ii) designing a consumer demands analysis procedure; and (iii) developing a system for analyzing consumer demands. This study tests the effectiveness of the computer-based approach for analyzing eWOM in the context of the Taiwanese fast food industry.

- The Influence of Information Overload on the Development of Trust and Purchase Intention Based on Online Product Reviews in a Mobile vs. Web Environment: An Empirical Investigation (by Christopher Paul Furner and Robert A. Zinko) recognizes that information overload has been studied extensively by decision science researchers, particularly in the context of task-based optimization decisions. Media selection research has similarly investigated the extent to which 
task characteristics influence media choice and use. This paper outlines a study which compares the effectiveness of web-based online product review systems for facilitation of trust and purchase intention to those of mobile product review systems in an experiential service setting (hotel services). Findings indicate that the extensiveness of information in the review increases trust and purchase intention until that information load becomes excessive, at which point trust and purchase intention begin to decrease. The magnitude of this decline is smaller in web-environments than in mobile environments, suggesting that web-based systems are more effective in fostering focus and are less prone to navigation frustration, thus reducing information overload.

- Exploring the Effects of Reward and Competition Intensity on Participation in Crowdsourcing Contest (by Dan Li and Longying $\mathrm{Hu}$ ) notes that with the growth of web 2.0 technology, Crowdsourcing contests offer task seekers a chance to gain access to expertise and resources with lower cost. Although many studies have investigated the effects of influencing factors on solvers' participation behavior, there has been scant research on the effect of com- petition intensity, as well as the relationships between competition and reward. Collecting data from Tasken website in China, this paper builds a two-equation model based on expectation-value theory to explore the effects of task reward and competition intensity on solvers' registration and submission behavior. The research results verify that task reward positively impacts the number of registrations and submissions, and competition intensity negatively affects solvers' submissions. In addition, the empirical results show that competition intensity moderates the relationship between task reward and submission. These findings provide valuable contributions to the crowdsourcing literature.

We sincerely hope that you enjoy this special issue.

Open Access This article is distributed under the terms of the Creative Commons Attribution 4.0 International License (http:// creativecommons.org/licenses/by/4.0/), which permits unrestricted use, distribution, and reproduction in any medium, provided you give appropriate credit to the original author(s) and the source, provide a link to the Creative Commons license, and indicate if changes were made. 